\title{
Artelogie
}

artelogie Recherche sur les arts, le patrimoine et la littérature de l'Amérique latine

$6 \mid 2014$

Horizons et dispositifs des arts plastiques des pays du Río de la Plata (XXe siècle)

\section{Clemente Padín, la subversion du mot et de l'objet}

\section{Elena Lespes Munoz}

\section{OpenEdition}

\section{Journals}

Édition électronique

URL : http://journals.openedition.org/artelogie/1286

DOI : 10.4000/artelogie.1286

ISSN : 2115-6395

\section{Éditeur}

Association ESCAL

Référence électronique

Elena Lespes Munoz, «Clemente Padín, la subversion du mot et de l'objet », Artelogie [En ligne],

6 | 2014, mis en ligne le 03 août 2017, consulté le 30 avril 2019. URL : http://journals.openedition.org/ artelogie/1286; DOI : 10.4000/artelogie.1286

Ce document a été généré automatiquement le 30 avril 2019

Association ESCAL 


\title{
Clemente Padín, la subversion du mot et de l'objet
}

\author{
Elena Lespes Munoz
}

Poète expérimental, artiste-postal, performeur, curateur, essayiste, Clemente Padín est un artiste uruguayen qui contribua activement, par ses revues, ses actions et ses différents manifestes, aux expérimentations artistiques menées par les artistes latinoaméricain dans le contexte trouble des dictatures. Le terme de "conceptualisme(s)», privilégié a posteriori par les historiens de l'art latino-américains, désigne, non pas un mouvement esthétique, mais ces formes hétéroclites prises par les réponses artistiques au contexte de censure et de répression de cette période. Aussi bien processuelles (performances, happening, photographies, vidéos, etc) que linguistiques (Nouvelle Poésie), elles ont en commun le rejet de l'institution Art et apparaissent comme indissociables des contextes dans lesquels elles prennent place. Le rejet des circuits et des langages traditionnels (musée, galerie ; peinture, sculpture, etc), et la dématérialisation de l'objet d'art, amènent les artistes à élaborer de nouvelles stratégies de circulation et de communication, de nouveaux langages et de nouvelles manières de faire de l'art. Le parcours de l'artiste Clemente Padín en est l'une des plus riches illustrations.

\section{Premières secousses}

2 En Uruguay, la radicalisation politique et artistique des années soixante est précipitée par le durcissement du régime et l'effondrement progressif de l'édifice démocratique. Le modèle politique et économique interventionniste de l'Etat, hérité du Batlisme ${ }^{1}$, est abandonné peu à peu au profit de la seule alternative libérale. Les taux d'inflation qui progressent à vive allure, pour atteindre 136\% en 1967, amènent le président Pacheco Areco (1967-1971) à instaurer une politique anti-inflationniste, qui, bien qu'efficace dans un premier temps, ne profite qu'aux grands propriétaires. Face à la crise de l'Etat intégrateur, le gouvernement fait face à un processus de radicalisation idéologique initié par les intellectuels et le corps professoral qui contamine le milieu syndical_. 
Durant les années 50 et 60 , la figure de «l'intellectuel engagé » est représentée par les

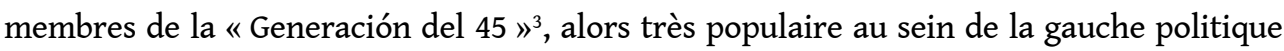
et du paysage littéraire latino-américain. Ce groupe constitué d'écrivains, de poètes et de

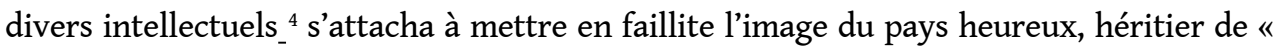
Como el Uruguay no hay" [Comme l'Uruguay, il n'y en a pas]. Le critique Angel Rama désignait le groupe, auquel il appartenait lui-même, par le terme de "Generación critica »" Ils dénonçaient la crise structurelle du pays et, dans un climat de polarisation politique et sociale, défendaient une "culture militante» en marge des partis politiques traditionnels :

Une culture qui ne croit (..) en aucune neutralité possible, mais qui en retour affirme la nécessité de récupérer avec lucidité et courage l'espace du réel dans lequel s'inscrit l'homme et qui croit en son action positive et transformatrice. (...) la culture doit être portée au cœur de la société, spécialement dans les secteurs sociaux les plus oubliés. ${ }_{-}^{6}$

Figure 1. Couverture Los Huevos del Plata $n^{\circ} 13$, mars 1969

\section{LOS HUeVoS del PLATA}

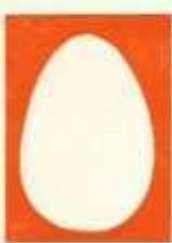

MARZO '69-No 13 MONTEVIDEO-URUGUAY

Sabés que aquí ahora se me ocurre que a la calle le está picando la nariz $\mathrm{Y}$ va a estornudar sabós che se me ocurre que de hace rato que jugamos con fuego y siempre terminamos haciéndonos pichí en la cama que se nos tapa la bombilla puteamos pero no cambiamos la yerba ni la bombilla ni el mate apenas escupimos algo verde sabés qué sońa. mos todas las noches $y$ al otros día jugamos al 48 y lo peor es que no sacamos nada de tanto mirar el sol vamos a tener que usar lentes los invito vamos $\alpha$ ver si se nos queman las alas se me ocurre no sé pero aqui en este momento si nos decidimos $\alpha$ soplar to dos juntos se va $\alpha$ levantar un viento que no va $a$ haber nube que se quede quieta sabés che se me ocurre que si jugamos $\alpha$ la mancha tiene que ser venenosa que cuando tiremos una baldosa lo hagamos bien fuerte cosa que recorra todas las calles se me ocurre no ś que capaz mañana cuando levantemos baldosas vamos $\alpha$ encontrar flores che por eso digo a la calle cada día le pica más la nariz y en cualquier momento puede estornudar $y$ ahí hermano vamos a tener que estar todos para

decirle salud eso si ese dia loco

se terminb la joda.

\section{ANIVERSARIO REVOLUCION CUBANA}

Archive Clemente Padín (UDELAR, Montevideo)

Avec le durcissement du régime, de jeunes auteurs et poètes _ expriment leur opposition à la "Generación del 45 ", soulignant l'écart entre l'engagement de ces intellectuels et la faiblesse de leurs actions ${ }^{8}$. Au travers de la revue Los Huevos del Plata, fondée en décembre 1965 par le poète Clemente Padín, ils tentèrent de développer un compromis alternatif entre culture et politique.

Marquée par la poésie expérimentale qui se développe alors en Amérique du Sud autour du concrétisme littéraire, en quête d'une plus grande participation du spectateur, la revue s'intéresse aux expérimentations menées par le groupe Poema/Processo (Brésil), au 
spatialisme de Pierre Garnier et aux recherches de Julien Blaine (France) et Edgardo Antonio Vigo (Argentine).

6 Le nom de la revue à double entente, se posait comme une réponse critique à celles d'un seul nom telles que Escritura ou Marcha; l'explicite sexuel porté sur la masculinité, transformait avec sarcasme la lutte pour les espaces de parole en action virile. En introduisant les auteurs du surréalisme, de la Beat Generation et les symbolistes français, Los Huevos del Plata s'émancipe d'une culture uruguayenne et latino-américaine régionaliste, revendiquant la désacralisation de l'expérience poétique et littéraire jusque dans sa référence assumée aux pays développés.

7 Sur la couverture du numéro de mars 1969, on peut lire la légende "100. Anniversario Revolución Cubana » sous un texte qui n'en fait pourtant pas mention; à l'intérieur du numéro d'octobre 1968, dédié à la commémoration du centenaire du Chant I de Maldoror, apparaît un bandeau noir avec la phrase "Gloria a los combatientes Liber Arce, Susana Pintos y Hugo de los Santos » [Gloire aux combattants...], trois jeunes communistes tués lors des manifestations de rues récentes 9 , à côté des analyses consacrées au poète né à Montevideo. Les membres de la revue, malgré une maîtrise des débats contemporains (la Révolution Cubaine, le Vietnam, Mai 68, la répression dans les rues de Montevideo...), se refusent à l'analyse et aux longs discours, à l'inverse de la gauche intellectuelle, accusée d'avoir "fait de la révolution, une montagne de papiers $»^{10}$. Selon eux, la culture ${ }^{11}$, et plus précisément l'art, doit avoir un impact immédiat sur la réalité. La démocratie déliquescente, marquée par l'imposition en 1968 des medidas prontas de seguridad ${ }^{12}$, contraint ces jeunes poètes à questionner l'apparente "vérité » du langage, alors instrument d'assujettissement social, en altérant les modes et les signes de communication. La revue, fondée sur un discours "politiquement" incorrect, iconoclaste et révolutionnaire, entame l'abandon progressif des avant-gardes historiques et inaugure des contre-canons littéraires qui seront pleinement développés dans la publication suivante, Ovum 10.

\section{La subversion des signes : le langage rendu objet}

8 La revue Ovum 10 ouvre une nouvelle voie par son articulation plus marquée entre les mouvements de résistance politique et les expériences radicales autour du langage. Lancée par le même Clemente Padín, la revue présente divers mouvements de la poésie expérimentale et poursuit une émancipation des circuits et des formes traditionnelles de l'art. Née en 1969, elle se développe parallèlement aux premières expositions consacrées à la Poésie Visuelle ${ }^{13}$ et aux nombreuses revues alternatives qui naissent sur l'ensemble du continent, Clemente Padín commence alors à réunir bon nombre d'entre elles : El Corno Emplumado (Mexique), El Caimàn 


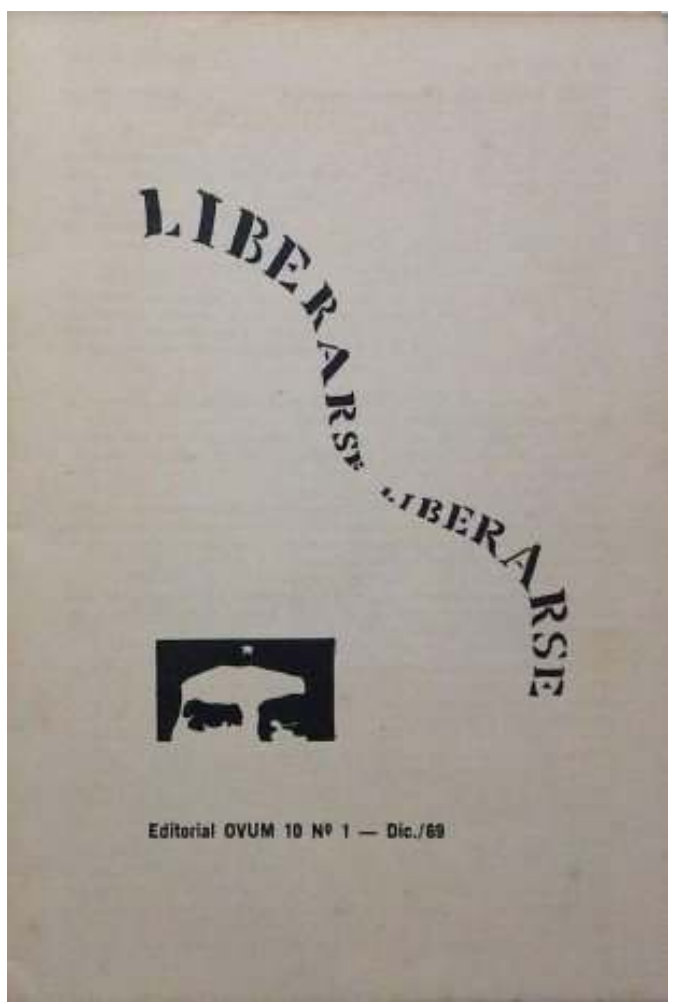

Archive Clemente Padín (UDELAR, Montevideo), Barbudo (Cuba), El Techo de la Ballena, La Plata de Palo (Venezuela), W.C, Diagonal Cero (Edgardo A. Vigo, Argentine), les Éditions Mimbre (Chili), etc.

9 La revue Ovum 10 est une revue essentiellement visuelle, pensée dès son principe pour dix numéros. L'éditorial du premier numéro, paru en décembre 1969, est un hommage à l'étudiant Liber Arce assassiné par la police un an plus tôt lors des manifestations étudiantes ${ }^{14}$. On constate que l'éditorial, place réservée traditionnellement dans les journaux à un mot du rédacteur en chef, est ici constitué d'un poème visuel non-signé ${ }^{15}$. Le mot «liberarse » [se libérer], par deux fois répété, produit une homonymie avec le nom de Liber Arce. L'ondulation typographique, doublée du sens de lecture, insuffle aux mots un mouvement de va-et-vient, qui est renforcé par le rétrécissement graphique au centre de la « phrase ». Ce rythme dissonant, comme un mot dont la première syllabe aurait été prononcée à voix haute et la seconde à voix basse et inversement, produit visuellement la sensation d'une parole en difficulté, en même temps que celle d'un sanglot. Dans la moitié inférieure de la page, une xylographie reproduit un fragment de la photographie du Che Guevara par Alberto Korda, cette confrontation avec le regard du célèbre libérateur, place la «phrase » sous le signe de l'injonction : «libérez-vous ! ». Ce cadrage sur les yeux du Che peut être lu dans un tout autre sens, dans la mesure où il évoque, paradoxalement, le regard instigateur du «Big Brother is watching you ». L'ondulation, drapeau flottant de la liberté par analogie, devient alors un effacement, une parole noyée : «liberarse » comme un mot dangereux à prononcer, «Liber Arce » comme ce mot tabou des premiers écueils d'une dictature en devenir. 


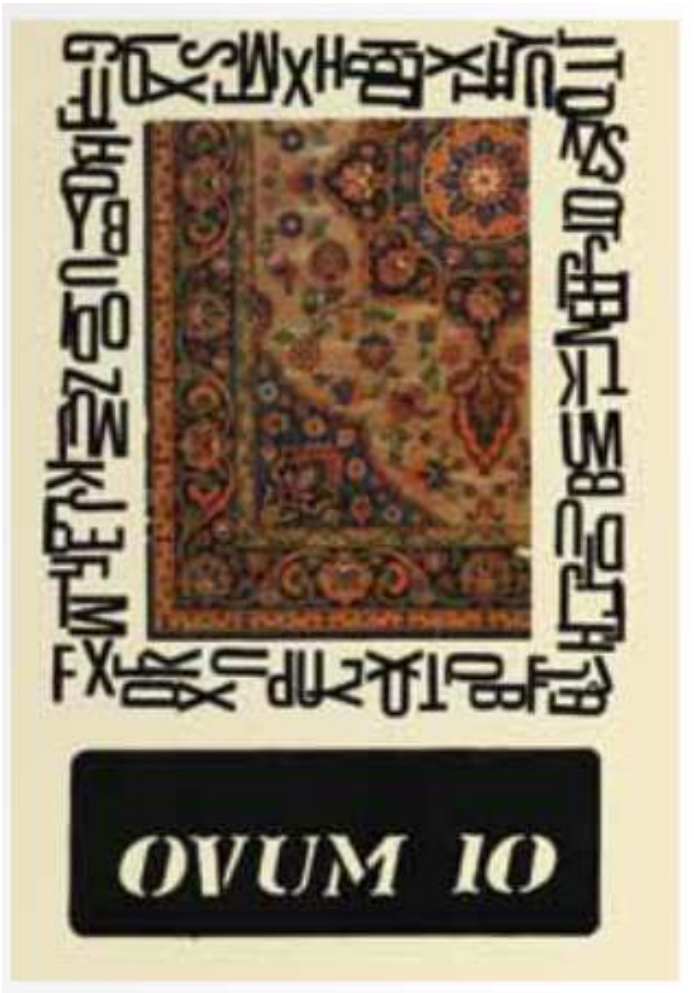

Archive Clemente Padín (UDELAR, Montevideo)

La parole empêchée devient le projet même du poète qui, place la déconstruction du langage au cœur du processus poétique, comme un acte radical en réponse à la violence imposée ${ }^{16}$. Dans son manifeste "La Nueva Poesia $»^{17}$, Clemente Padín revendique que soit redonnée au langage, sa nature d'objet, d'acte, et que prenne fin l'autorité de sa fonction expressive $^{18}$. Il a lu Saussure, selon qui le signe, un «terme en soi nul $»_{-}^{19}$, existe par association d'idées; c'est précisément cette historicité des formes que rejette le poète. L'accent porté sur la dimension matérielle des mots et des lettres participe de ce déplacement sémantique, non pas que le sens soit abandonné, mais plutôt se présente-t-il sous sa forme dématérialisée. La couverture du premier Ovum 10 présente une fraction de tapis oriental à l'intérieur d'un cadre de lettres. Les lettres forment une ligne continue et chaotique, un introuvable énoncé, montré-caché dans son désordre ornemental :

Beaucoup d'artistes de ma génération et moi-même assumions un type d'écriture qui ne paraissait pas laisser d'espace à 'la signification ' immédiate, une sorte de poésie asémantique. ${ }^{20}$

On peut interpréter cette disposition des lettres et de l'image de diverses façons : 1) La richesse des motifs du tapis n'est pas encadrée mais enfermée, comme le souvenir d'une poésie lyrique que l'on rangerait dans une boîte pour passer à autre chose, c'est la poésie expérimentale s'émancipant du cadre du Poème traditionnel ; 2) L'amas confus de lettres forme une ligne de démarcation, un fil barbelé de langage qui fait écho à la prolifération "végétale » des motifs du tapis, métaphore de la création. Le poète brésilien Ferreira Gullar, issu du néo-concrétisme brésilien, dans son texte "Théorie du Non-objet » datant de 1959 présente le «non-dit » comme l'unique source de la poésie qui se transmet au travers du «non-objet » : 
Le non-objet n'est pas un anti-objet mais c'est un objet spécial dans lequel on prétend réaliser une synthèse des expériences sensorielles et mentales : un corps transparent à la connaissance phénoménologique, intégralement perceptible, qui se laisse percevoir sans laisser de trace. Une pure apparence. ${ }^{21}$

Reprochant au Concrétisme, et particulièrement au Grupo Noigandres, un faire trop mathématique qui repose sur les relations mécaniques des mots entre eux, Ferreira Gullar ne prétend pas à la destruction du vers mais révise la syntaxe visuelle du poème. Le blanc de la page devient le silence rendu matériel, les espaces entre les mots ne sont plus des respirations, mais les suintements d'un silence iconique. Le silence de la page devient un espace symbolique qui amplifie le signifié. Pour Clemente Padín, les opérateurs sémantiques, phoniques et grammaticaux ne sont plus les éléments déterminant de la fonction poétique du langage, à ceux là s'ajoute l'opérateur visuel (le travail sur la lettre et l'espace qu'elle occupe) : il s'agit de transformer le mot, le symbole, en une figure, autrement dit une icône, par la projection de codes non-verbaux sur le code verbal ${ }^{22}$.

Figure 4. «PATRIA/PARIA », Jorge Caraballo, 1977

\section{PATR I A}

$\mathrm{PA}_{T} \mathrm{R}, \mathrm{A}$

PARIA

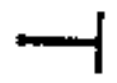

13 Le poème «PATRIA/PARIA » de Jorge Caraballo, poète uruguayen proche de Clemente Padín, repose sur une opposition lexicale et sur une homophonie partielle. "Patria» [patrie] dit l'attachement, l'appartenance natale, civique et nationale, l'inclusion. " Paria » dit l'exclusion, le rejet, Jorge Caraballo étant en prison lors de l'écriture de ce poème ${ }^{23}$, il désigne la figure de l'exilé. En tant que prisonnier politique, il se voit exclu de/par la patrie, mis au ban. Le parallélisme phonique est renforcé par la lettre T qui matérialise le passage entre l'appartenance et l'exclusion, la perte de la lettre $\mathrm{T}$ figure la perte de la patrie : la lettre est littéralement expulsée hors du mot, hors de la patrie. Les trois temps du poème se déroulent comme ceci: 1) le citoyen d'une patrie quelconque, 2) l'ébranlement du mot, la crise du concept de " patrie » par l'effondrement du consensus démocratique, 3) l'exclusion, la condition de paria a-patride. La chute de la lettre T, et 
non sa disparition, renforce son caractère d'opérateur visuel, passant du simple code linguistique (dans le mot "patrie») à un code visuel, voire iconique : l'exil intérieur comme une chute, comme une mise en croix.

14 Ces expérimentations poétiques radicales sont déplacées au sein de nouvelles structures et formes de communication qui menacent l'entropie du langage par l'importance qu'elles accordent à la circulation et à l'imprévisibilité des échanges.

\section{L'Art Postal : circulation et contre-information}

15 L'échange de travaux poétiques et artistiques par voie postale était une pratique fort répandue dans les années soixante: héritée du groupe Fluxus, on retrouve notamment cette pratique chez Robert Filliou dans son Étude d'acheminement de poèmes en petite vitesse et chez l'américain Ray Johnson, qui ouvrit en 1962 The New-York Correspondance School of Art. Cependant cette pratique acquiert un caractère distinct dans le contexte des dictatures et apparaît ici comme une poétique surgie de l'urgence. En effet, le rejet grandissant des institutions muséales d'une part, assujetties pour une grande partie aux gouvernements, et du réseau des galeries d'autre part, associé au système Art reposant sur la valeur marchande de l'objet artistique, engendre une série de stratégies de la part des artistes pour se libérer de ces contraintes, tout en leur assurant une visibilité ${ }^{4}$. L'art qui circule par voie postale est une pratique nécessitant peu de moyens et qui s'émancipe des catégories de l'auteur et de l'œuvre unique :

L'Art Postal déplace le projecteur de ce que l'on appelle traditionnellement l'œuvre d'art vers le concept plus large de culture. C'est ce changement qui fait que l'art postal est réellement contemporain. L'art postal emphatise des stratégies culturelles où les limites seraient celles entre le travail de l'artiste et l'organisation et la distribution comme structure de ce même travail. En ce sens, ces stratégies sont ses composantes formelles. ${ }^{25}$.

16 Ainsi, le réseau postal représente un circuit alternatif, où la manière même de faire de l'art est transformée: interaction, co-création, œuvre en processus ou œuvre comme processus, œuvre participative, etc. Les courants présents dans ce circuit sont extrêmement divers et rendent difficile la définition d'une œuvre d'Art Postal : Poésie Visuelle, néo-dadaïsme, constructivisme, registres de performances, consignes, manifestes, etc. Aussi variées soient-elles, ses pratiques se définissent cependant par un médium qui constitue une fin en soi :

Lorsque l'on envoi une sculpture par courrier, l'artiste se limite à utiliser un moyen de transport déterminé pour déplacer son œuvre, déjà élaborée. Au contraire, le nouveau langage artistique que nous sommes en train d'analyser repose sur le fait que la distance déterminée que doit parcourir l'œuvre, fait partie de sa structure, c'est l'œuvre. L'œuvre a été créée pour être envoyée par courrier et ce fait conditionne sa création (dimension, franchise, poids, nature du message, etc.). ${ }^{26}$

17 L'Art Postal repose alors sur trois critères pleinement réactivés par le médium: la communication, l'information et la circulation. Dans le contexte de censure des dictatures où l'information devient synonyme de propagande officielle, les notions de circulation et d'accès à l'information sont des enjeux fondamentaux. La revue Ovum publiée par Clemente Padín en 1972 suite à Ovum 10, est conçue à partir du réseau postal (revue coopérative) et diffusée uniquement par ce dernier.

18 Les fruits de ces correspondances rhizomiques étaient par ailleurs présentés dans le cadre d'expositions organisées par les artistes eux-mêmes : on peut citer le Festival de la Postal 
Creativa, organisé en 1974 par Clemente Padín à la Galerie U de Montevideo ou la II Exposição Internacional de Arte Postal au Brésil en 1976, à la suite de laquelle les organisateurs, Paulo Bruscky et Daniel Santiago, furent séquestrés par le régime.

Si le réseau postal reposait sur un principe démocratique (y participait qui voulait), le réseau des artistes, bien qu'étendu géographiquement, demeurait restreint numériquement (environ 200 participants). Les expositions et les revues étaient des lieux de visibilité et donc des moyens de luttes assumés comme tels, outre le fait que nombre des travaux qui circulaient à travers ce réseau étaient éminemment politiques.

Cette carte de Clemente Padín, relève d'un détournement explicitement politique d'une œuvre de Mondrian et fut envoyée par l'artiste à Walter Zanini comme participation à l'exposition Poeticas Visuais qui se tint en 1977 au Musée d'Art Contemporain de Sao Paulo ${ }^{27}$. On y voit la reproduction d'un fragment d'une œuvre de Piet Mondrian (est-ce véritablement la reproduction d'un fragment d'œuvre ou est-ce un fragment singé par Clemente Padín ?) : la première reproduction se présente

Figure 5. Clemente Padín, in catalogue Poeticas Visuais, 29 septembre - 30 octobre 1977,

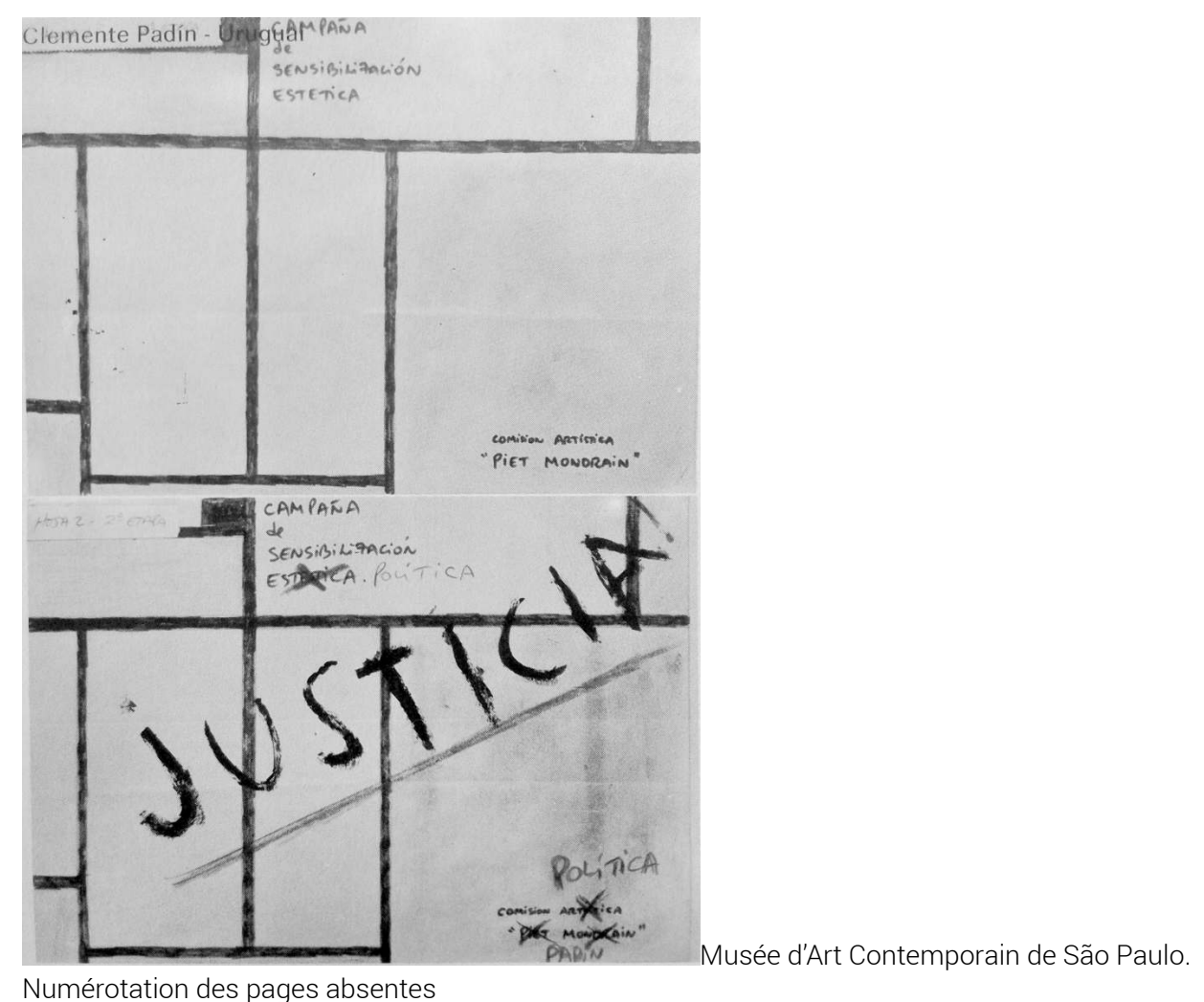

21 comme une "première étape ", on peut y lire "Clemente Padín - Campaña de sensibilización estetica " et "Comision artistica Piet Mondrain ${ }^{28}$ "; la seconde est semblable à l'exception du texte qui se trouve corrigé, on lit alors « Hoja 2 - 2nda etapa Campaña de sensibilización estetica política» et «Comision artistica Política Piet Mondrain Padín», le tout traversé en oblique par le mot «Justicia!» en lettres majuscules et souligné. Cette carte fut émise durant le boycott, auquel participa Clemente Padín, contre la section d'art latino-américaine instituée par la Xème Biennale de Paris. Les organisateurs de la Biennale avait confié cette section au gouvernement militaire uruguayen $^{29}$, qui désigna le directeur du Musée National d'Art Visuel de Montevideo. 
L'opposition à la Biennale, coordonnée par Felipe Erhenberg ${ }^{30}$, critiquait ce qui apparaissait comme une légitimation culturelle et politique du régime dictatorial uruguayen. Clemente Padín fut arrêté durant la nuit du 25 août 1977 et c'est sa participation au boycott de la Biennale qui fut le motif de son incarcération ${ }^{31}$. En 1980, Felipe Ehrenberg publia l'ensemble de la correspondance autour de cette action dans Expediente Bienal X (Mexique, Libre Acción Libre, Beau Geste Press). On y trouve une lettre de Clemente Padín qui lui est adressée, dont la signature et l'entête sont noircie et où l'on peut lire: "Photocopie originale d'un texte de l'uruguayen dont nous omettons les références personnelles pour des raisons compréhensibles ». Clemente Padín sortit de prison en 1979, mais demeura en liberté surveillée jusqu'en $1984^{32}$.

La force contestataire du réseau apparait évidente au vue de la réaction qu'elle engendre auprès de la censure et des régimes militaires, provoquant en retour de nombreuses campagnes internationales de soutient (campagne pour la libération de Clemente Padín et Jorge Caraballo, campagne contre la dictature du Paraguay, etc). L'Art Postal apparaît comme un réseau de contre-information, dans la mesure où les contenus véhiculés incitent à une attitude en totale rupture avec les régimes en place: rejet de l'individualisme, de la passivité, de la consommation, etc. Le caractère utopique, comme expression de l'espérance et de la responsabilité, de cette pratique, repose sur la construction d'une réalité dynamique, en-devenir. En effet, l'imprévisibilité de l'information esthétique est un élément clé de l'Art Postal, puisque la réception, la modification et les apports critiques engendrés par chaque échange sont constitutifs de l'œuvre en circulation :

L'Art Postal fonctionne alors comme une extension d'un corps social et politique émancipé de ses limites physiques. Un corps dont les frontières ne sont plus tracées par l'espace qu'il occupe, sinon par tous les espaces « virtuels » qu'il incarne. Un corps comme un dispositif qui peut se charger symboliquement de la même force disruptive que la présence. ${ }^{33}$ :

23 L'Art Postal accorde une place très importante aux pratiques performatives et participatives, dont il est le vecteur et la trace. Aussi, c'est très spontanément que Clemente Padín se tourne vers cette pratique et élabore un langage de l'action, nouvelle étape de sa pensée-manifeste.

\section{Poesia Inobjetal : pour un art sans objet}

Dans un contexte politique qui apparait comme chevillé, le rejet de l'attitude passive, conforté par le désir d'inclure le spectateur dans le processus créatif, répond à la nécessité d'agir sur le réel. L'action radicale prônée par les groupes de guérillas qui se développent dans nombre des pays du cône Sud, comme les Tupamaros en Uruguay, et présentée comme la seule réponse possible, influence bien évidemment les artistes qui se trouvent confrontés au problème de l'efficacité de l'art.

Dans la Poesia Inobjetal I, Clemente Padín poursuit sa réflexion sur le langage et met en cause son caractère mimétique, qui substitue à la réalité, une copie de celle-ci : 
Figure 6. INOBJETAL 1, Ovum 10, Montevideo, Avril 1971. Archives Clemente Padín (UDELAR, Montevideo)

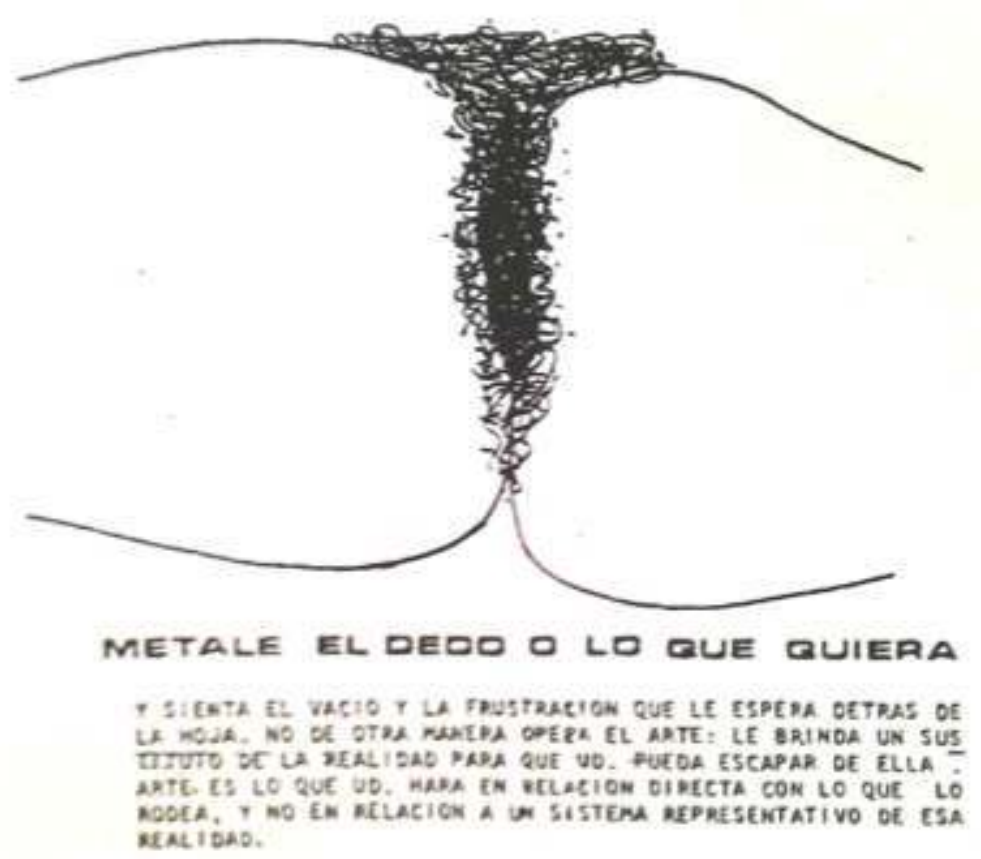

Mettez-lui un doigt ou ce que vous voudrez: Et sentez le vide et la frustration qui vous attendent derrière le vide de la feuille. L'art n'agit pas d'une autre façon : il offre un substitut à la réalité pour que vous puissiez lui échapper. Art, c'est ce que vous ferez en relation directe avec ce qui vous entoure, et non en relation avec un système représentatif de cette réalité. ${ }^{34}$.

Diffusés par voie postale, puis publié dans la revue Ovum 10, les manifestes INOBJETAL I, II, III et IV, synthétisent le projet de Clemente Padín sur le langage de l'action. La page blanche apparaît maintenant comme un espace limité qui doit être transcendé. L'acte doit être privilégié en tant qu'action déterminante de l'expression poétique. 


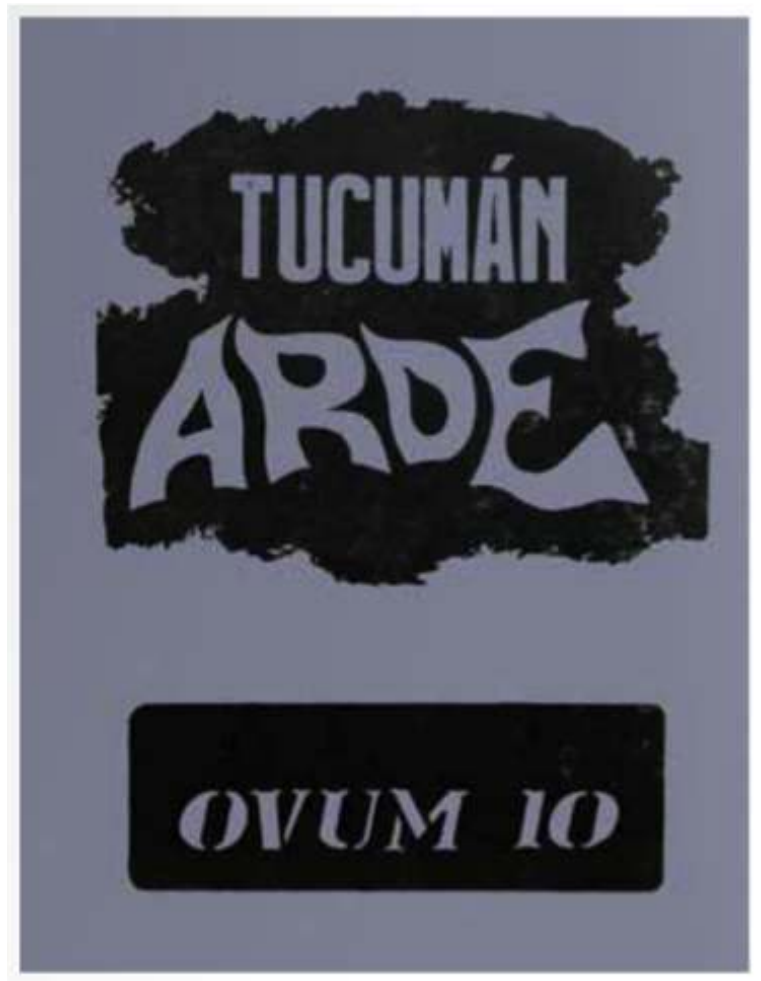

Clemente Padín consacre ainsi le numéro 9 de la revue Ovum 10, paru en 1971, à Tucuman Arde, élevée aujourd'hui en symbole de cette période de radicalisation artistique et politique. En 1968, en Argentine, des artistes de Rosario décidèrent, avec la CGT des Argentins (CGTA), de monter une opération de contre-information sur la situation de la province de Tucuman, région de l'industrie sucrière, alors en crise. Le désir de redéfinir les liens entre art et réalité, et art et politique (par l'association avec un groupe d'opposition), apparaît ici dans la volonté de diriger l'impact de la création artistique sur la vie sociale ${ }^{35}$. Clemente Padín prit connaissance de cette expérience au travers de l'article «Les Fils de Marx et Mondrian » paru dans le revue Robho $\left(n^{\circ} 5-6,1971\right)$ fondée par son ami Julien Blaine ${ }^{36}$. Lorsque Clemente Padín se rendit dans la ville de Rosario pour recueillir les témoignages de cette expérience pour sa publication, il ne retrouva aucun des participants, probablement parce que ces derniers avaient cessé toute activité artistique après cela.

Clemente Padín cherche donc à définir un langage de l'action, qui ouvrirait la voie à un réel malléable. Il aborde la question du langage en des termes marxistes, puisqu'il pointe du doigt, les liens de ce dernier avec l'idéologie : le langage, comme «système représentatif de la réalité ", est déterminé par son contenu idéologique, «il est possible d'utiliser les signes, de n'importe quel langage, pour déguiser la réalité d'un manteau de signaux $»^{37}$. La représentation du réel est un énoncé de nature sociale. 


\section{ANTES}
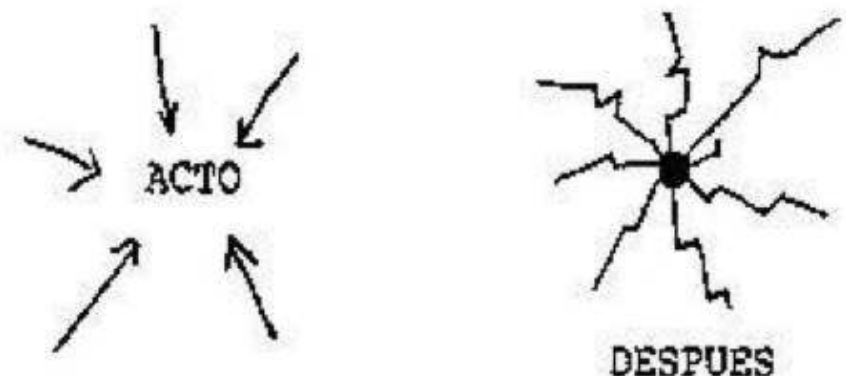

Archive Clemente Padín (UDELAR, Montevideo)

En cherchant à dépasser la dichotomie saussurienne langue/parole, il ré-affirme la nature objectale du signe, et donc sa nature sociale, pour poser les fondements d'une linguistique de l'action en tant que manifestation poético-sociale, individuelle ou collective. Le langage de l'action a pour signe l'acte, qui « au niveau du signifiant, ceuvre sur la réalité et, (...) au niveau du signifié, ceuvre idéologiquement $~_{38}$. L'acte-signe n'agit donc pas seulement au niveau idéologique mais directement sur le réel, puisqu'il permet au récepteur «plusieurs niveaux de compréhension de la réalité " $^{39}$ et c'est le devenir critique de cette compréhension qui fait œuvre.

INOBJETAL II se présentait sous la forme d'une feuille imprimée pliée en quatre et agrafée, sur laquelle on pouvait lire «Prohibido " [Défense d'ouvrir] en plusieurs langues. Une fois la feuille ouverte, le récepteur pouvait lire "Vous avez, en ne respectant pas l'interdiction, montré que vous aviez une personnalité indépendante et que désormais tout dépendait de vous, tout est possible alors... $\star^{40}$. L'œuvre commence avec l'intention de voir désobéir le récepteur, continue dans la désobéissance devenue effective, et se poursuit au-delà, dans les désobéissances à venir: l'acte-signe fonctionne en quelque sorte comme un acte initiatique, autrement dit «l'art doit se sortir de l'art »".

Il est intéressant de constater que très souvent, le caractère esthétique des pratiques engendrées au travers de l'Art Postal et à partir de ces différents manifestes était secondaire :

Nous considérions ces œuvres comme des instruments de dénonciation. C'était autre chose de les considérer pour elles-mêmes, ou pour leurs qualités esthétiques. En vérité, c'était des œuvres d'art; mais pour nous c'était une catégorisation secondaire. En premier lieu, ce qui réellement importait, c'était combien elles pouvaient influer sur les gens qui entraient en contacts avec ces travaux. ${ }^{42}$. 
Figure 9. Registre photographique, Clemente Padín, Por el arte y por la paz, durant l'inauguration de l'exposition « America Latina, Hoy, 1984 », Galerie DAAD, 21 mai 1984.
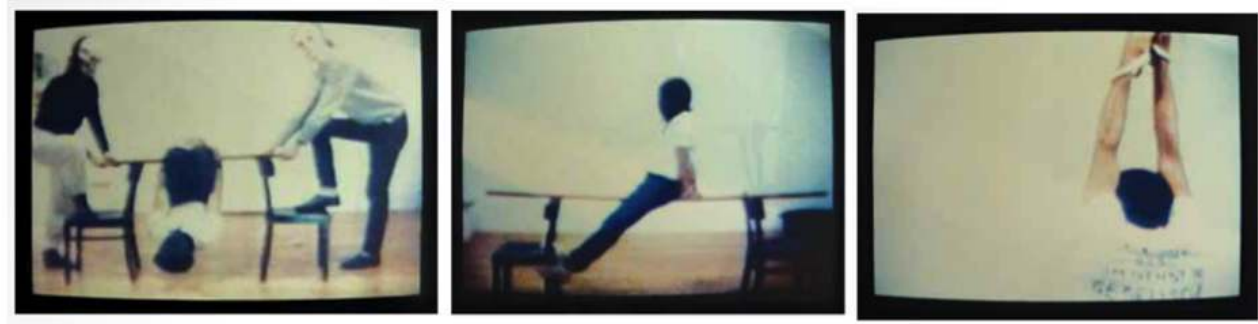

Archive Clemente Padín (UDELAR, Montevideo)

Lorsqu'en 1984, Clemente Padín obtient une bourse de l'Ambassade d'Allemagne, il organise une exposition sur l'Art Postal latino-américain, intitulée "America Latina, hoy, 1984 » à la Galerie DAAD située dans Berlin Ouest et dirigée par René Block. Le 21 mai 1984, jour de l'inauguration, il réalise la performance Por el arte y la paz, qui consista en un inventaire, mimé, des mécanismes de torture durant la lecture d'un manifeste. Plus qu'une œuvre d'art, elle représentait un acte de dénonciation: Clemente Padín tenait à rappeler le contexte dans lequel s'inscrivait la pratique de l'Art Postal, d'autant qu'en 1984 , la dictature uruguayenne n'avait pas encore pris fin.

La dureté du régime, les bouleversements sociaux et la mise en «crise » des institutions artistiques ont participés à l'émergence d'un «art » confronté aux limites de sa propre définition, comme en témoigne Clemente Padín, dans son hésitation à faire du critère esthétique, un élément déterminant de ses pratiques. Le caractère utopique d'une telle démarche transparaît dans le souci d'articuler la déconstruction du langage traditionnel à la conception d'un langage de l'action, autrement dit dans le souci de dépasser la distinction art/vie, voire de la nier. La conservation sous forme d'archives des expériences menées par Clemente Padín (Archives Clemente Padín, UDELAR, Montevideo) révèle le caractère hybride de ces documents difficiles à définir (œuvres, traces, fragments, documentations, etc). Présentés comme des morceaux de « vie », ils s'exposent aujourd'hui comme une tentative de réactiver ce temps de l'utopie, au présent.

\section{BIBLIOGRAPHIE}

Arte contemporáneo brasileño: documentos y críticas, coord. Glória Ferreira, Saint-Jacques de Compostelle, Artedardo, 2009.

BENVENUTO Luis y otros, Uruguay hoy, Buenos Aires, Siglo XXI, 1971.

BRUSCKY Paulo, «Arte Correio e a grande rede : Hoje, a arte é este comunicado », 1976 (retravaillé en 1981 et 2005).

FERNANDA Nogueira, «El cuerpo político más allá de sus límites. Clemente Padín y el flujo postal », paru dans Artecorreo, México DF, Museo de la Ciudad de México; RM Verlag, 2011, pp 77-92.

Disponible sur http://fernandanogueira-escritos.blogspot.fr/ 
FREIRE Cristina et LONGONI Ana (sous la direction de), Conceitualismos do Sul-Sur, Sao Paulo, édition Annablume, 2008.

FREIRE Cristina, Poéticas do processo : arte conceitual no museu, São Paulo, Iluminuras, 1999.

LONGONI Ana et MESTMAN Mariano, Del Di Tella a « Tucuman Arde ", in Vanguardia artística y política en el 68 argentino, Université de Buenos-Aires, Eudeba, 2008.

Los Huevos del Plata n¹0, décembre 1967.

Los Huevos del Plata $\mathrm{N}^{\circ} 12$, octobre 1968.

Los Huevos del Plata ${ }^{\circ}$ 14, novembre 1969.

Marcha, 12 décembre 1965, consulté sur http://biblioteca.periodicas.edu.uy/, le 17/01/14.

PADín Clemente, La Poesia Experimental Latinoamericana, Collection Ensayo n²1, Información y

Producciones, s.l

PADín Clemente, «La Nueva Poesia y las redes alternativas », in interview avec Fernando DAVIS et Fernanda NOGUEIRAS, ERRATA \#, Revista de Artes Visuales, « La escritura del arte » n², août 2010

SAUSSURE F. de, Écrits de linguistique générale, Paris, Gallimard, 2002

\section{NOTES}

1. Le modèle "battliste" repose sur une redistribution partielle des bénéfices du commerce extérieur et sur le développement de l'État-Providence. Il est né de l'impulsion du président José Battle y Ordoñez (1903-1907 et 1911-1915), qui instaura une politique sociale très avancée (législation du travail, politique éducative généralisée, système étendu de sécurité sociale) et une intervention active de l'état dans la vie économique et politique du pays (nationalisation de la Banque de la République, etc).

2. Les longues marches des cañeros, les ouvriers de la canne à sucre (1962, 1964, 1968 et 1971) jusqu'à la capitale rythment cette période et ont un rôle déterminant dans l'engagement de la jeunesse urbaine.

3. Connue également sous le nom de la «Génération des revues » ou « de Marcha », en référence à la fondation du séminaire « Marcha » par Carlos Quijano en 1939.

4. Parmi lesquels on peut citer Mario Benedetti, José Pedro Diaz, Carlos Maggi, Amanda Beranguer, Carlos Real de Azua...

5. Voir Ángel Rama, "La generación crítica", en Benvenuto, Luis y otros, Uruguay hoy, Buenos Aires: Siglo XXI, 1971.

6. Ángel Rama, « La cultura militante », Marcha, 12 décembre 1965.

7. Héctor Paz, Julio Moses, Juan José Linares et Clemente Padín. Le groupe se désagrégea peu à peu et seul Clemente Padín poursuivit le travail initié.

8. Nombre des membres de la "Generación del 45 " occupaient désormais une chaire à l'université et eurent pour élèves les fondateurs de Los Huevos del Plata.

9. Los Huevos del Plata, $\mathrm{n}^{\circ} 12$, octobre 1968.

10. Los Huevos del Plata, $n^{\circ} 10$, décembre 1967.

11. Ils iront jusqu'à l'appeler la «Gran Puta » dans le dernier numéro de la revue, Los Huevos del Plata, $\mathrm{n}^{\circ} 14$, novembre 1969.

12. La censure et les arrestations arbitraires sont banalisées.

13. La première, «Liberarse ", à l'Ancienne Faculté des Sciences Humaines de l'Université de la République à Montevideo en 1969. Autrement, on peut citer, « Exposicion exhaustiva de la Nueva 
Poesia » en 1972 la Galerie U de Montevideo, dirigée par Enrique Gómez. Toutes deux furent organisées par Clemente Padín.

14. Le 9 août 1968, l'autonomie universitaire est violée par le gouvernement qui ordonne la fouille des locaux des facultés d'Agronomie, d'Architecture, de Médecine et des Beaux-Arts de l'Université de la République en quête de preuves contre le non-respect des medidas prontas des seguridas. L'événement déclenche une série de manifestations étudiantes.

15. On sait cependant qu'il est de Clemente Padín.

16. Suite à son élection en 1971, le président Juan Maria Bordaberry mit en place une « dictature constitutionnelle ", les mesures exceptionnelles de sécurité (article 168) furent appliquées durant une durée exceptionnelle (36 mois sur 49 mois de régime). En avril 1972, le parlement déclara l' «état de guerre interne » suite à l'assassinat par les Tupamaros du sous-secrétaire d'Etat à l'intérieur Armando Costa y Lara. Répression, censure et emprisonnement politique étaient de mise.

17. Ovum 10, n²1, décembre 1969.

18. Clemente Padín, in Freire, Cristina et Longoni, Ana (sous la direction de), Conceitualismos do Sul-Sur, édition Annablume, Sao Paulo, 2008, pp. 225-226.

19. Saussure, F. de, in Écrits de linguistique générale, Paris, Gallimard, 2002, p. 109.

20. Clemente Padín dans une interview avec Fernando Davis et Fernanda Nogueira, in ERRATA \#, Revista de Artes Visuales, «La escritura del arte » n², août 2010, p. 196.

21. Ferreira Gullar, «Teoria do Não-objeto », Rio de Janeiro : Jornal do Brasil, Suplemento dominical, 20 décembre 1959. Reproduit dans Arte contemporáneo brasileño: documentos y críticas, coord. Glória Ferreira, Artedardo, Saint-Jacques de Compostelle, 2009.

22. Clemente Padín, in La Poesia Experimental Latinoamericana, Collection Ensayo $n^{\circ} 1$, Información y Producciones s.l,p. 215.

23. Clemente Padín et Jorge Caraballo ont tous deux été détenus par la police dans la nui du 25 août 1977.

24. «L' Art Postal (Mail Art), Art par Correspondance, Art à Domicile ou quelle que soit la dénomination qu'il reçoit, n'est pas un «isme» de plus, sinon la solution la plus viable qui existait pour l'art de ces dernières années et les raisons en sont simples : anti-bougeois, anticommercial, anti-système, etc. ", Paulo Bruscky, in « Arte Correio e a grande rede : Hoje, a arte é este comunicado", 1976 (retravaillé en 1981 et 2005). Consulté le 30 avril 2014, sur http:// www.festivaldearte.fafcs.ufu...

25. Ulíses Carrion, cité par Cristina Freire, in Poéticas do processo : arte conceitual no museu, Freire C., Iluminuras, São Paulo, 1999.

26. Horacio Zabala et Edgardo Antonio Vigo, in «Arte Correio : uma nova forma de expressao », cité par Paulo Bruscky, op. cit.

27. Walter Zanini, directeur du MAC-USP, invita des artistes du monde entier à lui envoyer un travail par voie postale pour cette exposition.

28. On peut se demander si le nom de Piet Mondrian n'a pas été mal orthographié volontairement.

29. Le 27 juin 1973, l'armée a proclamé la dissolution du Congrès et du Parlement, Bordaberry, maintenu à son poste, instaura une dictature, s'auto-proclamant Proceso de reconstrucción nacional, sous la surveillance étroite d'un Conseil d'Etat formé de militaires. La Constitution fut suspendue, les partis politiques interdits et les mouvements sociaux violemment réprimés. En juin 1976, l'armée destitua Bordaberry, Alberto Demicheli lui succéda avant d'être remplacé par Aparicio Mendez en septembre de la même année.

30. Artiste mexicain, membre du Grupo Processo Pentagono.

31. Et non son activité dans le Parti Communiste (distribution de tracts) ou ses activités avec Ovum, même si certaines des publications servirent de preuves pour sa condamnation pour 
«escarnio y vilipendio moral de las Fuerzas Armadas » [Outrage et mépris à l'encontre des Forces Armées].

32. Confiscation du passeport, interdiction de quitter Montevideo et d'exercer un emploi, rendez-vous de contrôle hebdomadaire, correspondance interdite, etc.

33. Fernanda Nogueira, «El cuerpo político más allá de sus límites. Clemente Padín y el flujo postal ", in Artecorreo, México DF, Museo de la Ciudad de México; RM Verlag, 2011, pp 77-92. Disponible sur http://fernandanogueira-escritos.blogspot.fr

34. INOBJETAL I: Metale el dedo o lo que quiera, in Ovum 10, avril 1971, Montevideo. Reproduit dans La Poesia Experimental Latinoamericana, op. cit., p. 205.

35. L'opération se déroula en plusieurs temps: deux campagnes d'affichage dans la ville de Rosario, un voyage de recherches dans la province de Tucuman et la restitution de cette investigation dans le cadre d'une «exposition» dans les locaux de la CGTA de Rosario, puis de Buenos-Aires. Pour plus d'information sur le sujet voir : Longoni, Ana et Mestman Mariano, Del Di Tella a "Tucuman Arde»,Vanguardia artística y política en el 68 argentino, Eudeba, Université de Buenos-Aires, 2008.

36. Il peut apparaître surprenant que Clemente Padín n'ait eu connaissance de cette expériences qu'à travers l'article d'une revue française, mais il nous faut tenir compte des aléas de la communication entre les pays d'Amérique du Sud durant cette période, cela malgré l'importance du réseau de l'Art Postal

37. Clemente Padín, "El lenguaje de la acción », publié en Mai 1975 dans la revue Abertura Cultural de Rio de Janeiro, Brésil. Reproduit dans La Poesia Experimental Latinoamericana, op.cit. , p. 190

38. Ibid, p. 190.

39. Ibid, p. 191.

40. INOBJETAL II, Mai 1971. Ibid, pp. 193-194.

41. INOBJETAL IV, ibid, p.196.

42. Clemente Padín dans une interview avec Fernando Davis et Fernanda Nogueira, op. cit., p. 209.

\section{RÉSUMÉS}

Durant les années 1960 et 1970, nombre de pays d'Amérique du Sud sont gouvernés par des dictatures militaires. Dans ce contexte politique qui a de profondes répercussions sur la vie sociale et culturelle, les artistes sont amenés à repenser leurs pratiques. La perte de confiance dans les institutions culturelles, la remise en cause des formes traditionnelles de l'art et la nécessité de se confronter au réel apparaissent comme des enjeux fondamentaux. Cet article présente ces bouleversements au travers de l'œuvre de l'artiste uruguayen Clemente Padín.

Durante los años sesenta y setenta, varios países de América del Sur viven bajo una dictadura. Frente a estas tensiones políticas que impactan violentamente la vida social y cultural, los artistas tratan de repensar sus practicas artísticas. La perdida de fe en las instituciones culturales, el replanteamiento de las formas tradicionales del arte y la necesidad por impactar la realidad aparecen como problemáticas fundamentales de este periodo. El presente articulo trata de estas alteraciones a través de la obra del artista uruguayo Clemente Padín. 
INDEX

Palabras claves : Uruguay, dictadura, Clemente Padín, poesía experimental, arte postal

Mots-clés : Uruguay, dictature, Clemente Padín, poésie expérimentale, art postal

\section{AUTEUR}

\section{ELENA LESPES MUNOZ}

Université Panthéon-Sorbonne/Université de São Paulo, Brésil 